\title{
On the determinant bundles of abelian schemes
}

\author{
Vincent Maillot and Damian Rössler
}

\begin{abstract}
Let $\pi: \mathcal{A} \rightarrow S$ be an abelian scheme over a scheme $S$ which is quasi-projective over an affine noetherian scheme and let $\mathcal{L}$ be a symmetric, rigidified, relatively ample line bundle on $\mathcal{A}$. We show that there is an isomorphism

$$
\operatorname{det}\left(\pi_{*} \mathcal{L}\right)^{\otimes 24} \simeq\left(\pi_{*} \omega_{\mathcal{A}}^{\vee}\right)^{\otimes 12 d}
$$

of line bundles on $S$, where $d$ is the rank of the (locally free) sheaf $\pi_{*} \mathcal{L}$. We also show that the numbers 24 and $12 d$ are sharp in the following sense: if $N>1$ is a common divisor of 12 and 24 , then there are data as above such that

$$
\operatorname{det}\left(\pi_{*} \mathcal{L}\right)^{\otimes(24 / N)} \not \mathcal{\sim}\left(\pi_{*} \omega_{\mathcal{A}}^{\vee}\right)^{\otimes(12 d / N)} .
$$
\end{abstract}

\section{Introduction}

Let $\pi: \mathcal{A} \rightarrow S$ be a abelian scheme, where $S$ is a scheme that is quasi-projective over an affine noetherian scheme. We denote as usual by $\omega_{\mathcal{A}}$ the determinant of the sheaf of differentials of $\pi$. Let $\mathcal{L}$ be a line bundle on $\mathcal{A}$. Let $\epsilon: S \rightarrow \mathcal{A}$ be the zero-section and suppose that the line bundle $\epsilon^{*} \mathcal{L}$ is the trivial line bundle. Suppose furthermore that there is an isomorphism $[-1]^{*} \mathcal{L} \simeq \mathcal{L}$ and that $\mathcal{L}$ is ample relative to $\pi$. In this situation, Faltings and Chai prove the following result (see [FC90, Theorem 5.1, p. 25]).

Theorem 1.1 (Chai-Faltings). There is an isomorphism $\operatorname{det}\left(\pi_{*} \mathcal{L}\right)^{\otimes 8 d^{3}} \simeq\left(\pi_{*} \omega_{\mathcal{A}}^{\vee}\right)^{\otimes 4 d^{4}}$ of line bundles on $S$.

Here $d$ is the rank of the (locally free) sheaf $\pi_{*} \mathcal{L}$. This is a refinement of a special case of the formule clé considered by Moret-Bailly in his monograph [MB85].

In [FC90, p. 27], Faltings and Chai state that it is nevertheless likely that the factor $d^{3}$ can be cancelled on both sides of the above isomorphism, or in other words that it is likely that there is an isomorphism

$$
\operatorname{det}\left(\pi_{*} \mathcal{L}\right)^{\otimes 8} \simeq\left(\pi_{*} \omega_{\mathcal{A}}^{\vee}\right)^{\otimes 4 d} .
$$

Let us introduce the line bundle

$$
\Delta(\mathcal{L}):=\operatorname{det}\left(\pi_{*} \mathcal{L}\right)^{\otimes 2} \otimes \pi_{*} \omega_{\mathcal{A}}^{\otimes d}
$$

The existence of the isomorphism (1) is the statement that $\Delta(\mathcal{L})^{\otimes 4}$ is trivial.

The aim of this paper is to present the proof of the following statements about $\Delta(\mathcal{L})$.

THEOREM 1.2 .

(a) There is an isomorphism $\Delta(\mathcal{L})^{\otimes 12} \simeq \mathcal{O}_{S}$.

Received 6 March 2007, accepted in final form 22 May 2007, published online 14 March 2008.

2000 Mathematics Subject Classification 14K15, 14K25, 14C40.

Keywords: determinant bundles, abelian schemes, key formula, Adams-Riemann-Roch.

This journal is (C) Foundation Compositio Mathematica 2008. 


\section{Maillot AND D. RÖSSleR}

(b) For every $g \geqslant 1$, there exist data $\pi: \mathcal{A} \rightarrow S$ and $\mathcal{L}$ as above such that $\operatorname{dim}(\mathcal{A} / S)=g$ and such that $\Delta(\mathcal{L})$ is of order 12 in the Picard group of $S$.

The following corollary follows immediately from Theorem 1.2(a) and Theorem 1.1.

Corollary 1.3. If $(3, d)=1$ then $\Delta(\mathcal{L})^{\otimes 4}$ is trivial.

Notice that Theorem 1.2(b) in particular implies that the exponent 4 surmised by Faltings and Chai is not the right one (it has to be replaced by the exponent 12). The corollary says that the exponent 4 is nevertheless the right one when $(3, d)=1$.

The fact that $\Delta(\mathcal{L})$ is a torsion line bundle is a consequence of the Grothendieck-RiemannRoch theorem. This was shown by Moret-Bailly and Szpiro in Appendix 2 to Moret-Bailly's monograph [MB85] and also by Chai in his thesis (see [Chai85, ch. V, paragraph 3, Theorem 3.1, p. 209]). The link between the Grothendieck-Riemann-Roch theorem and the fact that $\Delta(\mathcal{L})$ is a torsion line bundle was already known to Mumford in the early 1960s (private communication between Chai and the authors). If $S$ is a smooth quasi-projective scheme over $\mathbb{C}$, then Theorem 1.2(a) is contained in a theorem of Kouvidakis (see [Kou00, Theorem A]). The method of proof of the theorem of Kouvidakis is analytic and is based on the study of the transformation formulae of theta functions. It extends earlier work by Moret-Bailly (see [MB90]), who considered the case where $d=1$. The result of Kouvidakis was extended by Polishchuk to more general bases $S$ in [Pol00]. Polishchuk's proof is a refinement of Faltings and Chai's proof of Theorem 1.1; this last proof is not based on the Riemann-Roch theorem. The Theorem 0.1 in [Pol00] shows in particular that there exists a constant $N(g)$, which depends only on the relative dimension $g$ of $\mathcal{A}$ over $S$, such that $\Delta(\mathcal{L})^{\otimes N(g)}$ is trivial. Theorems $0.1,0.2$ and 0.3 of [Pol00] give various bounds for $N(g)$, which depend on $d$, on $g$ and on the residue characteristics of $S$. In this context, the content of Theorem 1.2 is that, $N(g)=12$ is a possible choice and that, for each $g \geqslant 1$, it is the best possible choice.

A key input in Polishchuk's refinement of the proof of Faltings and Chai is a formula describing the behaviour of $\Delta(\mathcal{L})$ under isogenies of abelian schemes ([Pol00, Theorem 1.1]; see also the end of $\S 2$ ), which generalises an earlier formula by Moret-Bailly (see [MB85, VIII, 1.1.3, p. 188]), who considered the case $d=1$. The proof of Theorem 1.2(a) presented in this paper combines Polishchuk's isogeny formula and a refinement of the Grothendieck-Riemann-Roch theorem, called the Adams-Riemann-Roch theorem (see $\S 2$ ). In spirit, it is close to Mumford's original approach. Our method can also be related to Moret-Bailly's proof of the formule clé in positive characteristic; see the first remark at the end of the text. Our proof of Theorem 1.2(b) is based on a lemma of Polishchuk and on two constructions of Mumford.

The plan of the paper is as follows. The second section contains some preliminaries to the proof; these preliminaries are the Adams-Riemann-Roch theorem and the two results of Polishchuk mentioned in the last paragraph. The proof itself is contained in the third section.

Notation and conventions. Suppose that $\mathcal{M}$ is a line bundle on a group scheme $\mathcal{C}$ over a base $B$, with zero-section $\epsilon: B \rightarrow \mathcal{C}$. We shall say that $\mathcal{M}$ is rigidified if $\epsilon^{*} \mathcal{M}$ is the trivial line bundle. We shall say that $\mathcal{M}$ is symmetric if $[-1]^{*} \mathcal{M} \simeq \mathcal{M}$. Suppose that $x$ is an element of an abelian group $G$ and that $k$ is a positive integer; we shall say that $x$ is a $k^{\infty}$-torsion element of $G$ if there exists an integer $n \geqslant 0$ such that $k^{n} \cdot x=0$ in $G$. If $G$ is a group or a group functor and $k$ is a strictly positive integer, we shall write $[k]$ for the map $G \rightarrow G$ such that $[k](x)=x+\cdots+x$ (k times) for every $x \in G$.

\section{Preliminaries}

In this section, 'scheme' will be short for 'noetherian scheme'. 


\section{ON THE DETERMINANT BUNDLES OF ABELIAN SCHEMES}

\subsection{The Adams-Riemann-Roch theorem}

In this section, we first describe the special case of the Adams-Riemann-Roch theorem that we shall need. We then go on to describe Polishchuk's isogeny formula.

If $Y$ is a scheme, we shall write as usual $K_{0}(Y)$ for the Grothendieck group of coherent locally free sheaves. The tensor product of locally free sheaves descends to a bilinear pairing on $K_{0}(Y)$, which makes it into a commutative ring. If $f: X \rightarrow Y$ is a morphism of schemes, the pull-back of $\mathcal{O}_{Y}$-modules induces a ring morphism $f^{*}: K_{0}(Y) \rightarrow K_{0}(X)$. As a ring, $K_{0}(Y)$ is endowed with a family $\left(\psi^{k}\right)_{k \in \mathbb{N}^{*}}$ of (ring) endomorphisms, called the Adams operations. They have the property that $\psi^{k}(\mathcal{M})=\mathcal{M}^{\otimes k}$ in $K_{0}(Y)$ for every line bundle $\mathcal{M}$ on $Y$. Furthermore, if $f: X \rightarrow Y$ is a scheme morphism as before, then $f^{*} \circ \psi^{k}=\psi^{k} \circ f^{*}$. The Adams operations are uniquely determined by these two last properties and by the fact that they are ring endomorphisms.

We shall also need Bott's 'cannibalistic' classes. We shall denote thus a family of operations $\left(\theta^{k}\right)_{k \in \mathbb{N}^{*}}$, each of which associates elements of $K_{0}(Y)$ to coherent locally free sheaves on $Y$. They have the following three properties, which determine them uniquely. For every line bundle $\mathcal{M}$ on $Y$, we have

$$
\theta^{k}(\mathcal{M})=1+\mathcal{M}+\mathcal{M}^{\otimes 2}+\cdots+\mathcal{M}^{\otimes(k-1)}
$$

in $K_{0}(Y)$. If

$$
0 \rightarrow E^{\prime} \rightarrow E \rightarrow E^{\prime \prime} \rightarrow 0
$$

is an exact sequence of coherent locally free sheaves on $Y$, then $\theta^{k}\left(E^{\prime}\right) \theta^{k}\left(E^{\prime \prime}\right)=\theta^{k}(E)$. Finally, if $f: X \rightarrow Y$ is a morphism of schemes, then $f^{*}\left(\theta^{k}(E)\right)=\theta^{k}\left(f^{*} E\right)$, for every coherent locally free sheaf on $Y$. The following lemma holds about the operations $\theta^{k}$. Suppose for the time of the lemma that $Y$ is quasi-projective over an affine scheme.

Lemma 2.1. For any coherent locally free sheaf $E$ on $Y$, the element $\theta^{k}(E)$ is invertible in the ring $K_{0}(Y)[1 / k]$.

Proof. See [Ros99, paragraph 4, Proposition 4.2] (for lack of a standard reference).

Let $f: X \rightarrow Y$ be a flat and projective morphism of schemes. We may consider the Grothendieck group $K_{0}^{\text {ac }}(X)$ of $f$-acyclic coherent locally free sheaves on $X$, i.e. coherent locally free sheaves $E$ such that $R^{i} f_{*} E=0$ for every $i>0$. There is a unique morphism of groups $f_{*}: K_{0}^{\operatorname{ac}}(X) \rightarrow$ $K_{0}(Y)$ such that $f_{*}(E)=R^{0} f_{*} E$ for every coherent locally free sheaf on $X$. A theorem of Quillen (see [Qui72, paragraph 4, Theorem 3, p. 108]) now implies that the natural map $K_{0}^{\text {ac }}(X) \rightarrow K_{0}(X)$ is an isomorphism. Hence we obtain a morphism $f_{*}: K_{0}(X) \rightarrow K_{0}(Y)$. This morphism satisfies the projection formula: for all $y \in K_{0}(Y)$ and all $x \in K_{0}(X)$, the identity $f_{*}\left(f^{*}(y) \otimes x\right)=y \otimes f_{*}(x)$ holds.

Let us now consider a smooth and projective morphism of schemes $f: X \rightarrow Y$, where $Y$ is quasiprojective over an affine scheme. Let $\Omega$ be the sheaf of differentials associated to $f$; it is a locally free sheaf on $X$. In this situation, the Adams-Riemann-Roch theorem is the following statement.

Theorem 2.2 (Grothendieck et al.). For any $x \in K_{0}(X)[1 / k]$, the equality

$$
\psi^{k}\left(f_{*}(x)\right)=f_{*}\left(\theta^{k}(\Omega)^{-1} \psi^{k}(x)\right)
$$

holds in $K_{0}(Y)[1 / k]$.

For a proof of the Adams-Riemann-Roch theorem, see [FL85, V, paragraph 7, Theorem 7.6, p. 149].

\subsection{Some results of Polishchuk on $\Delta(\mathcal{L})$}

Let $T$ be any base scheme and let $\kappa: \mathcal{B} \rightarrow T$ be an abelian scheme. Let $\alpha: \mathcal{B} \rightarrow \mathcal{B}$ be a finite and flat $T$-homomorphism of group schemes. Let $\mathcal{M}$ be a symmetric and rigidified line bundle on $\mathcal{B}$, 


\section{Maillot AND D. RÖSSleR}

which is ample relative to $\kappa$. Suppose that the sheaf $\kappa_{*} \mathcal{M}$ has strictly positive rank. The following special case of Polishchuk's isogeny formula [Pol00, Theorem 1.1] plays a crucial role in the proof of Theorem 1.2(a).

TheOrem 2.3 (Polishchuk).

(a) Let $n:=(12, \operatorname{deg}(\alpha))$. There is an isomorphism

$$
\operatorname{det}\left(\kappa_{*}\left(\alpha^{*} \mathcal{L}\right)\right)^{\otimes 2 n} \simeq \operatorname{det}\left(\kappa_{*}(\mathcal{L})\right)^{\otimes(2 n \cdot \operatorname{deg}(\alpha))} .
$$

(b) Let $m:=(3, \operatorname{deg}(\alpha))$. Suppose that $\operatorname{deg}(\alpha)$ is odd and that $\operatorname{rk}\left(\kappa_{*} \mathcal{L}\right)$ is even. There is then an isomorphism

$$
\operatorname{det}\left(\kappa_{*}\left(\alpha^{*} \mathcal{L}\right)\right)^{\otimes m} \simeq \operatorname{det}\left(\kappa_{*}(\mathcal{L})\right)^{\otimes(m \cdot \operatorname{deg}(\alpha))}
$$

The two following lemmas are needed in the proof of Theorem 1.2(b).

Lemma 2.4 (Polishchuk). Suppose that $\operatorname{dim}(\mathcal{B} / T)=1$ and that $\mathcal{M}=\mathcal{O}\left(O_{\mathcal{B}}\right) \otimes \omega_{\mathcal{B}}$. Then for every $r \geqslant 1$, there is an isomorphism

$$
\Delta\left(\mathcal{M}^{\otimes r}\right) \simeq \omega_{\mathcal{B}}^{\otimes\left(r^{2}+2\right)}
$$

Here $O_{\mathcal{B}}$ is the image of the unit section of $\mathcal{B} / T$. Notice that the image of the unit section is a Cartier divisor and that its normal bundle is isomorphic to the restriction of $\omega_{\mathcal{B}}$ via the unit section (this is a consequence of the fact that $\kappa$ is smooth; see for instance [FL85, IV, paragraph 3, Lemma 3.8]). This implies that the pull-back of $\mathcal{M}$ via the unit section is the trivial line bundle (see [FL85, IV, paragraph 3, Proposition 3.2(b)]).

For the proof of Lemma 2.4, see [Pol00, Proposition 5.1].

Let now $\kappa^{\prime}: \mathcal{B}^{\prime} \rightarrow T$ be an abelian scheme and let $\mathcal{M}^{\prime}$ be a symmetric and rigidified line bundle on $\mathcal{B}^{\prime}$, which is ample relative to $\kappa^{\prime}$. Let $p$ (respectively $p^{\prime}$ ) be the natural projection $\mathcal{B} \times{ }_{B} \mathcal{B}^{\prime} \rightarrow \mathcal{B}$ $\left(\right.$ respectively $\mathcal{B} \times_{B} \mathcal{B}^{\prime} \rightarrow \mathcal{B}^{\prime}$ ). Let $m$ (respectively $m^{\prime}$ ) be the rank of $\kappa_{*} \mathcal{M}$ (respectively $\kappa_{*}^{\prime} \mathcal{M}^{\prime}$ ).

LEMma 2.5. There is an isomorphism

$$
\Delta\left(p^{*} \mathcal{M} \otimes p^{\prime *} \mathcal{M}^{\prime}\right) \simeq \Delta(\mathcal{M})^{\otimes m^{\prime}} \otimes \Delta\left(\mathcal{M}^{\prime}\right)^{\otimes m} .
$$

Proof. This is left to the reader (use the Künneth formula).

\section{The proof}

\subsection{The isomorphism $\Delta(\mathcal{L})^{\otimes 12} \simeq \mathcal{O}_{S}$}

In this section, we shall prove assertion (a) in Theorem 1.2. We shall now apply the AdamsRiemann-Roch theorem (Theorem 2.2) to abelian schemes. We work in the situation of the Introduction. We may also suppose without restriction of generality that $d \geqslant 1$. Let $k \geqslant 2$. Let $g$ be the relative dimension of $\mathcal{A}$ over $S$. Recall that the theorem of the cube (see [MB85, paragraph 5.5, p. 29]) implies that $[k]^{*} \mathcal{L} \simeq \mathcal{L}^{\otimes k^{2}}$. Write $\Omega$ for the sheaf of differentials of $\pi$. We compute in $K_{0}(S)[1 / k]$ :

$$
\begin{aligned}
\psi^{k^{2}}\left(\pi_{*} \mathcal{L}\right) & =\pi_{*}\left(\theta^{k^{2}}(\Omega)^{-1} \psi^{k^{2}}(\mathcal{L})\right)=R \pi_{*}\left(\theta^{k^{2}}(\Omega)^{-1} \mathcal{L}^{\otimes k^{2}}\right) \\
& =\pi_{*}\left(\theta^{k^{2}}(\Omega)^{-1}[k]^{*}(\mathcal{L})\right)=\pi_{*}\left([k]^{*} \mathcal{L}\right) \theta^{k}\left(\pi_{*} \Omega\right)^{-1}
\end{aligned}
$$

where we have used the theorem of the cube, the projection formula and the fact that $\pi^{*} \pi_{*} \Omega=\Omega$. In other words, we have the identity

$$
\theta^{k^{2}}\left(\pi_{*} \Omega\right) \psi^{k^{2}}\left(\pi_{*} \mathcal{L}\right)=\pi_{*}\left([k]^{*} \mathcal{L}\right)
$$




\section{ON THE DETERMINANT BUNDLES OF ABELIAN SCHEMES}

in $K_{0}(S)[1 / k]$. Let us now introduce the (truncated) Chern character

$$
\operatorname{ch}: K_{0}(S)[1 / k] \rightarrow \mathbb{Z}[1 / k] \oplus \operatorname{Pic}(S)[1 / k],
$$

which is defined by the formula

$$
\operatorname{ch}\left(s / k^{t}\right):=\operatorname{rank}(s) / k^{t} \oplus \operatorname{det}(s)^{1 / k^{t}}
$$

for every $s \in K_{0}(S)$ and $t \in \mathbb{N}$. Let us introduce the pairing

$$
\left(r / k^{t}, m / k^{l}\right) \bullet\left(\left(r^{\prime}\right)^{1 / k^{t^{\prime}}},\left(m^{\prime}\right)^{1 / k^{l^{\prime}}}\right):=r \cdot r^{\prime} / k^{t+t^{\prime}} \oplus\left(m^{\prime}\right)^{r / k^{t+l^{\prime}}} \otimes m^{r^{\prime} / k^{t^{\prime}+l}}
$$

in the group $\mathbb{Z}[1 / k] \oplus \operatorname{Pic}(S)[1 / k]$. The pairing $\bullet$ makes this group into a commutative ring. The properties of the determinant show that the Chern character is a ring morphism. We now apply the Chern character to the identity (2). As we shall compute in the $\operatorname{ring} \mathbb{Z}[1 / k] \oplus \operatorname{Pic}(S)[1 / k]$, we switch from multiplicative notation $(\otimes)$ to additive notation $(+)$ in the group $\operatorname{Pic}(S)$. For the purposes of computation, we may suppose without loss of generality that $\pi_{*} \Omega=\omega_{1}+\cdots+\omega_{g}$ in $K_{0}(S)$, where $\omega_{1}, \ldots, \omega_{g}$ are line bundles. We compute

$$
\begin{aligned}
\operatorname{ch}\left(\theta^{k^{2}}\left(\pi_{*} \Omega\right)\right) & =\left(k^{2}+\frac{k^{2}\left(k^{2}-1\right)}{2} \operatorname{det}\left(\omega_{1}\right)\right) \bullet \cdots \bullet\left(k^{2}+\frac{k^{2}\left(k^{2}-1\right)}{2} \operatorname{det}\left(\omega_{g}\right)\right) \\
& =k^{2 g}+\frac{k^{2}\left(k^{2}-1\right) k^{2 g-2}}{2} \operatorname{det}\left(\pi_{*} \Omega\right)
\end{aligned}
$$

and

$$
\begin{aligned}
\operatorname{ch}\left(\theta^{k^{2}}\left(\pi_{*} \Omega\right)\right) \operatorname{ch}\left(\psi^{k^{2}}\left(\pi_{*} \mathcal{L}\right)\right) & =\left(k^{2 g}+\frac{k^{2}\left(k^{2}-1\right) k^{2 g-2}}{2} \operatorname{det}\left(\pi_{*} \Omega\right)\right) \bullet\left(d+k^{2} \operatorname{det}\left(\pi_{*} \mathcal{L}\right)\right) \\
& =k^{2 g} d+k^{2 g+2} \operatorname{det}\left(\pi_{*} \mathcal{L}\right)+\frac{d k^{2}\left(k^{2}-1\right) k^{2 g-2}}{2} \operatorname{det}\left(\pi_{*} \Omega\right) .
\end{aligned}
$$

On the other hand, we have

$$
\operatorname{ch}\left(\pi_{*}\left([k]^{*} \mathcal{L}\right)\right)=d k^{2 g}+\operatorname{det}\left(\pi_{*}[k]^{*} \mathcal{L}\right) .
$$

Here we have used the fact that the degree of the isogeny given by multiplication by $k$ on $\mathcal{A}$ is $k^{2 g}$ and the fact that the rank of $\pi_{*}[k]^{*} \mathcal{L}$ is $d k^{2 g}$ (see [Mum70, III, paragraph 12, Theorem 2, p. 121]). Thus, (2) leads to the equality

$$
k^{2 g} d+k^{2 g+2} \operatorname{det}\left(\pi_{*} \mathcal{L}\right)+\frac{d k^{2}\left(k^{2}-1\right) k^{2 g-2}}{2} \operatorname{det}\left(\pi_{*} \Omega\right)=d k^{2 g}+\operatorname{det}\left(\pi_{*}[k]^{*} \mathcal{L}\right)
$$

in $\mathbb{Z}[1 / k] \oplus \operatorname{Pic}(S)[1 / k]$. Multiplying by $k^{-2 g}$ and specialising to $\operatorname{Pic}(S)[1 / k]$, we get

$$
k^{2} \operatorname{det}\left(\pi_{*} \mathcal{L}\right)+\frac{d\left(k^{2}-1\right)}{2} \operatorname{det}\left(\pi_{*} \Omega\right)=k^{-2 g} \operatorname{det}\left(\pi_{*}[k]^{*} \mathcal{L}\right)
$$

in $\operatorname{Pic}(S)[1 / k]$. Now Theorem 2.3(a) shows that

$$
2 \cdot k^{-2 g} \operatorname{det}\left(\pi_{*}[k]^{*} \mathcal{L}\right)=2 \cdot \operatorname{det}\left(\pi_{*} \mathcal{L}\right)
$$

in $\operatorname{Pic}(S)[1 / k]$. We deduce from the last two equalities that

$$
\left(k^{2}-1\right) \cdot\left(2 \cdot \operatorname{det}\left(\pi_{*} \mathcal{L}\right)+d \cdot \operatorname{det}\left(\pi_{*} \Omega\right)\right)=0
$$

in $\operatorname{Pic}(S)[1 / k]$. In other words, $\Delta(\mathcal{L})^{\otimes\left(k^{2}-1\right)}$ is a $k^{\infty}$-torsion line bundle. If we specialise to $k=2$, we see that $\Delta(\mathcal{L})^{\otimes 3}$ is a $2^{\infty}$-torsion line bundle. If we specialise to $k=3$, we see that $\Delta(\mathcal{L})^{\otimes 8}$ is a $3^{\infty}$-torsion line bundle. Hence $\Delta(\mathcal{L})^{\otimes 24}$ is a trivial line bundle.

Suppose now that $d$ is odd. Theorem 1.1 says that $\Delta(\mathcal{L})^{\otimes 4 d^{3}}$ is a trivial line bundle. Hence $\Delta(\mathcal{L})^{\otimes\left(24,4 d^{3}\right)}$ is a trivial line bundle. Since $\left(24,4 d^{3}\right)$ divides 12 , this implies that $\Delta(\mathcal{L})^{\otimes 12}$ is a trivial line bundle. 


\section{Maillot AND D. RÖSSleR}

Suppose now that $d$ is even. Theorem 2.3(b) then shows that the equality

$$
k^{-2 g} \operatorname{det}\left(\pi_{*}[k]^{*} \mathcal{L}\right)=\operatorname{det}\left(\pi_{*} \mathcal{L}\right)
$$

holds in $\operatorname{Pic}(S)[1 / k]$ (this equality refines (3)). Proceeding as we did after the equality (4), we obtain the equality

$$
\frac{k^{2}-1}{2}\left(2 \cdot \operatorname{det}\left(\pi_{*} \mathcal{L}\right)+d \cdot \operatorname{det}\left(\pi_{*} \Omega\right)\right)=0
$$

in $\operatorname{Pic}(S)[1 / k]$. In other words, $\Delta(\mathcal{L})^{\otimes\left(k^{2}-1\right)}$ is a $k^{\infty}$-torsion line bundle if $k$ is even and $\Delta(\mathcal{L})^{\otimes\left(k^{2}-1\right) / 2}$ is a $k^{\infty}$-torsion line bundle if $k$ is odd. If we specialise to $k=3$, we see that $\Delta(\mathcal{L})^{\otimes 4}$ is a $3^{\infty}$-torsion line bundle. We saw above that $\Delta(\mathcal{L})^{\otimes 3}$ is a $2^{\infty}$-torsion line bundle and so we obtain again that $\Delta(\mathcal{L})^{\otimes 12}$ is a trivial line bundle.

This concludes the proof of the assertion (a) of Theorem 1.2.

Remark 1. Suppose that $S$ is a scheme over $\mathbb{F}_{p}$, for some prime number $p$ and that $d=1$. MoretBailly then proves that the line bundle $\Delta(\mathcal{L})^{\otimes\left(p^{2}-1\right) p^{2 g+2}}$ is trivial (see [MB85, ch. VIII, paragraph 2, Theorem 2.1, p. 193]). The equality (4) for $k=p$ is a variant of this. Notice furthermore that, for any vector bundle $E$ on $S$, we have $\psi^{p}(E)=F_{S}^{*}(E)$, where $F_{S}$ is the absolute Frobenius endomorphism of $S$. Moret-Bailly's proof is based on the study of the behaviour of $\Delta(\mathcal{L})$ under base change by $F_{S}$ and on the case $d=1$ of the isogeny formula. In this sense, our proof of (2) over a general base can be considered as an extension of Moret-Bailly's proof of (2) in positive characteristic.

\subsection{Sharpness}

In this section, we shall prove the assertion (b) in Theorem 1.2. We fix an affine noetherian base scheme $B$. All schemes and morphisms of schemes in this section will be relative to this base scheme. Furthermore, all schemes will be locally noetherian. We first recall a result of Mumford. Consider the following set of data:

(i) $\delta, g \in \mathbb{N}^{*}$;

(ii) $T$ a scheme;

(iii) $\kappa: \mathcal{B} \rightarrow T$ a projective abelian scheme of relative dimension $g$;

(iv) $\lambda: \mathcal{B} \rightarrow \mathcal{B}^{\vee}$ a polarisation over $T$ of degree $\delta^{2}$;

(v) a linear rigidification $\mathbb{P}\left(\kappa_{*}\left(L^{\Delta}(\lambda)^{\otimes 3}\right)\right) \simeq \mathbb{P}_{T}^{6^{g} \cdot \delta-1}$.

Here $L^{\Delta}(\lambda)$ is the pull-back of the Poincaré line bundle on $\mathcal{B} \times_{T} \mathcal{B}^{\vee}$ via the map $\operatorname{Id} \times_{T} \lambda: \mathcal{B} \rightarrow$ $\mathcal{B} \times_{T} B^{\vee}$. We shall call the scheme $T$ the ground scheme of the set of data. If we are given two sets of data as above, there is an obvious notion of isomorphism between them. If we are given two sets of data with the same ground scheme $T$, an isomorphism between the two sets of data will be called a $T$-isomorphism if it restricts to the identity on $T$. For each scheme $T$, we shall write $\mathcal{H}_{g, \delta}(T)$ for the set of $T$-isomorphism classes of sets of data whose ground scheme is $T$. If $T^{\prime} \rightarrow T$ is a morphism of schemes, the obvious base change of sets of data from $T$ to $T^{\prime}$ induces a map $\mathcal{H}_{g, \delta}(T) \rightarrow \mathcal{H}_{g, \delta}\left(T^{\prime}\right)$. One thus obtains a contravariant functor from the category of (locally noetherian) schemes to the category of sets. For more details, see [MFK94, ch. 7, paragraph 2].

Theorem 3.1 (Mumford). The functor $\mathcal{H}_{g, \delta}$ is representable by a quasi-projective scheme over $B$.

For the proof, see [MFK94, Proposition 7.3, ch. 7, paragraph 2]. We shall refer to the scheme representing $\mathcal{H}_{g, \delta}$ as $H_{g, \delta}$.

Let now $\kappa_{1,1}: \mathcal{B}_{1,1} \rightarrow H_{1,1}$ be the universal abelian scheme over $H_{1,1}$. Let

$$
\mathcal{L}_{\mathcal{B}_{1,1}}:=\mathcal{O}\left(O_{\mathcal{B}_{1,1}}\right)^{\otimes 3} \otimes \omega_{\mathcal{B}_{1,1} / H_{1,1}}^{\otimes 3}
$$




\section{ON THE DETERMINANT BUNDLES OF ABELIAN SCHEMES}

Here again $O_{\mathcal{B}_{1,1}}$ is the image of the unit section of $\mathcal{B}_{1,1} \rightarrow H_{1,1}$.

Proposition 3.2. If $B=\operatorname{Spec} \mathbb{C}$ then the line bundle $\Delta\left(\mathcal{L}_{\mathcal{B}_{1,1}}\right)$ is of order 12 in $\operatorname{Pic}\left(H_{1,1}\right)$.

Proof. Notice that there is a natural action of the group scheme $\mathrm{PGL}_{6}$ on $H_{1,1}$, defined as follows. Consider a set of data $\mathfrak{D}$ of the type described at the beginning of the section; let $a \in \mathrm{PGL}_{6}(T)$; to $a$ corresponds by construction an automorphism $A$ of $\mathbb{P}_{T}^{5}$; we let $a$ send $\mathfrak{D}$ on the set of data $\mathfrak{D}$ with its linear rigidification composed with $A$. This defines an action of the group functor $\mathrm{PGL}_{6}$ on the functor $\mathcal{H}_{1,1}$ and hence an action of $\mathrm{PGL}_{6}$ on $H_{1,1}$. Since $H_{1,1}$ is a fine moduli space, this $\mathrm{PGL}_{6}$-equivariant structure canonically lifts to a $\mathrm{PGL}_{6}$-equivariant structure on $\mathcal{B}_{1,1}$, such that the morphism $\kappa_{1,1}$ is $\mathrm{PGL}_{6}$-equivariant.

Let now $k \geqslant 1$ be the order of $\Delta\left(\mathcal{L}_{\mathcal{B}_{1,1}}\right)$ in $\operatorname{Pic}\left(H_{1,1}\right)$ (which is finite by Theorem 1.2(a)). Notice that Lemma 2.4 shows that there is an isomorphism

$$
\Delta\left(\mathcal{L}_{\mathcal{B}_{1,1}}\right) \simeq \kappa_{1,1 *} \omega_{\mathcal{B}}^{11}
$$

We thus see that there is an isomorphism

$$
\kappa_{1,1 *} \omega_{\mathcal{B}_{1,1}}^{\otimes 11 \cdot k} \simeq \mathcal{O}
$$

Fix such an isomorphism. This is tantamount to giving a trivialising section $s$ of $\kappa_{1,1 *} \omega_{\mathcal{B}_{1,1}}^{\otimes 1 \cdot k}$. Notice now that the reduced closed subscheme $H_{1,1 \text {,red }}$ underlying $H_{1,1}$ carries a $\mathrm{PGL}_{6}$-action such that the closed immersion $H_{1,1, \text { red }} \hookrightarrow H_{1,1}$ is equivariant (this follows from the definition of a group-scheme action, from [Gro60, I, paragraph 5, Corollary 5.1.8] and from the fact that $\mathrm{PGL}_{6} \times H_{1,1}$ is reduced, since $\mathrm{PGL}_{6}$ is smooth over $\mathbb{C}$ ). Furthermore, there are no non-trivial characters PGL $_{6} \rightarrow \mathbb{G}_{m}$. Thus the restriction of the section $s$ to $H_{1,1, \text { red }}$ is $\mathrm{PGL}_{6}$-invariant (for this, see [MFK94, Proposition 1.4, ch. I, paragraph 3, p. 33]).

Now consider an elliptic curve $\kappa: E \rightarrow$ Spec $\mathbb{C}$, which has complex multiplication by $\mathbb{Z}[j]$, where $j=-1 / 2+i \sqrt{3} / 2$ is a primitive third root of unity. The element $j$ then acts on $\kappa_{*} \omega_{E}$ by multiplication by either $j$ or $\bar{\jmath}$ (this can be seen by considering the complex uniformisation of $E(\mathbb{C}))$. Choose an arbitrary rigidification of $\mathbb{P}\left(\kappa_{*}\left(\mathcal{O}\left(O_{E}\right)^{\otimes 3}\right)\right)$. The elliptic curve $E$ together with its rigidification defines an element $P$ of $H_{1,1, \text { red }}(\mathbb{C})$, since Spec $\mathbb{C}$ is reduced. Since the section $s$ is PGL $_{6}$-invariant on $H_{1,1, \text { red }}$, the element $s(P) \in \kappa_{*} \omega_{E}^{\otimes 11 \cdot k}$ must satisfy the equation $s(P)=j^{11 \cdot k} \cdot s(P)$ or the equation $s(P)=\bar{\jmath}^{11 \cdot k} \cdot s(P)$ and hence $3 \mid k$. A similar reasoning with an elliptic curve with complex multiplication by the Gaussian integers $\mathbb{Z}[i]$ shows that $4 \mid k$. Hence 12 divides $k$; on the other hand Theorem 1.2(a) shows that $k$ divides 12 . Hence $k=12$ and this concludes the proof.

Remark 2. The idea to use rigidifications in the context of Theorem 1.1 is due to Faltings and Chai; see [FC90, proof of Theorem 5.1]. The idea to use elliptic curves with complex multiplication to compute orders in Picard groups is due to Mumford; see [Mum63, paragraph 6].

We shall now prove Theorem 1.2(b). Let $g$ be a positive natural number. Let $B=$ Spec $\mathbb{C}$ and choose an elliptic curve $E$ over $\mathbb{C}$. Let $\mathcal{L}_{E}:=\mathcal{O}\left(O_{E}\right)$ be the line bundle associated to the zero-section. Consider $E$ as an isotrivial abelian scheme over $H_{1,1}$. Consider the abelian scheme $\mathcal{A}:=\mathcal{B}_{1,1} \times H_{1,1} E^{g-1}$ over $S=H_{1,1}$ and the line bundle $\mathcal{L}:=\mathcal{L}_{\mathcal{B}_{1,1}} \otimes \mathcal{L}_{E}^{\otimes(g-1)}$ on $\mathcal{A}$ (here $\nabla$ refers to the exterior tensor product). It is a consequence of Proposition 3.2 and Lemma 2.5 that $\Delta(\mathcal{L})$ is of exact order 12 in $\operatorname{Pic}(S)$.

\section{ACKNOWLEDGEMENTS}

The authors wish to thank J.-B. Bost for interesting e-mail exchanges related to the properties of the bundle $\Delta(\mathcal{L})$. They are grateful to G. Faltings for explaining some aspects of the proof of 


\section{ON THE DETERMINANT BUNDLES OF ABELIAN SCHEMES}

Theorem 1.1 and to C.-L. Chai for reading an early version of the text and for providing them with various bibliographical and historical references. The authors thanks also go to the referee for his very useful suggestions. Finally, they would like to thank Ben Moonen for encouraging them to look for the proof of the fact that Theorem 1.2(a) is sharp.

\section{REFERENCES}

Chai85 C.-L. Chai, Compactification of Siegel moduli schemes, London Mathematical Society Lecture Note Series, vol. 107 (Cambridge University Press, Cambridge, 1985).

FC90 G. Faltings and C.-L. Chai, Degeneration of abelian varieties, with an appendix by David Mumford, Ergebnisse der Mathematik und ihrer Grenzgebiete (3), vol. 22 (Springer, Berlin, 1990).

FL85 W. Fulton and S. Lang, Riemann-Roch algebra, Grundlehren der mathematischen Wissenschaften, vol. 277 (Springer, Berlin, 1985).

Gro60 A. Grothendieck, Éléments de géométrie algébrique, Publ. Math. Inst. Hautes Études Sci. 4, 8, 11, 17, 20, 24, 28, 32 (1960-1967).

Kou00 A. Kouvidakis, Theta line bundles and the determinant of the Hodge bundle, Trans. Amer. Math. Soc. 352 (2000), 2553-2568.

MB85 L. Moret-Bailly, Pinceaux de variétés abéliennes, Astérisque 129 (1985), 266 pp.

MB90 L. Moret-Bailly, Sur l'équation fonctionnelle de la fonction thêta de Riemann, Compositio Math. 75 (1990), 203-217.

Mum63 D. Mumford, Picard groups of moduli problems, in arithmetical algebraic geometry, Proc. Conf. Purdue Univ., 1963 (Harper \& Row, New York, 1963), 33-81.

Mum70 D. Mumford, Abelian varieties, Tata Institute of Fundamental Research Studies in Mathematics, vol. 5 (Oxford University Press, London, 1970).

MFK94 D. Mumford, J. Fogarty and F. Kirwan, Geometric invariant theory, third edition, Ergebnisse der Mathematik und ihrer Grenzgebiete (2), vol. 34 (Springer, Berlin, 1994).

Pol00 A. Polishchuk, Determinant bundles for abelian schemes, Compositio Math. 121 (2000), 221-245.

Qui72 D. Quillen, Higher algebraic K-theory I, Lecture Notes in Mathematics, vol. 341 (Springer, Berlin, 1972).

Ros99 D. Rössler, An Adams-Riemann-Roch theorem in Arakelov geometry, Duke Math. J. 96 (1999), $61-126$.

Vincent Maillot vmaillot@math.jussieu.fr

Institut de Mathématiques de Jussieu, Université Paris 7 Denis Diderot, C.N.R.S., Case Postale 7012, 2 place Jussieu, F-75251 Paris cedex 05, France

Damian Rössler dcr@math.jussieu.fr

Institut de Mathématiques de Jussieu, Université Paris 7 Denis Diderot, C.N.R.S., Case Postale 7012, 2 place Jussieu, F-75251 Paris cedex 05, France 